\title{
The Effectiveness of Silodosin for Nocturnal Polyuria in Elderly Men With Benign Prostatic Hyperplasia: A Multicenter Study
}

\author{
${ }^{1}$ Department of Urology, Chungbuk National University College of Medicine, Cheongju, Korea \\ ${ }^{2}$ Department of Urology, Eulji University School of Medicine, Daejeon, Korea \\ ${ }^{3}$ Department of Urology, Konkuk University School of Medicine, Chungju, Korea \\ ${ }^{4}$ Department of Urology, Konyang University College of Medicine, Daejeon, Korea \\ ${ }^{5}$ Department of Urology, Yonsei University Wonju College of Medicine, Wonju, Korea
}

Young Won Kim ${ }^{1}$, Jinsung Park ${ }^{2}$, Hong Chung ${ }^{3}$, Hong-Wook Kim ${ }^{4}$, Hyung Joon Kim ${ }^{4}$, Jae Hung Jung ${ }^{5}$, Won Tae Kim ${ }^{1}$

Purpose: To investigate improvement in nocturia and nocturnal polyuria in nocturnal polyuria patients after silodosin administration by using a 3-day frequency volume chart.

Methods: This was a prospective multicenter study. We enrolled nocturnal polyuria patients (nocturnal polyuria index [NPi] $>0.33$ ), aged $\geq 60$ years, diagnosed with the 3 -day frequency volume charts of patients with benign prostatic hyperplasia taking a-blockers. Of the 54 patients, 30 (55.6\%) completed the study according to the study protocol (per-protocol group), and 24 dropped out (dropout group).

Results: Of the 24 patients in the dropout group, 5 withdrew consent due to side effects or lack of efficacy, 7 were lost to follow-up at 4 weeks, 8 were lost to follow-up at 12 weeks, and 4 dropped out due to failure to complete 3-day frequency volume charts at 12 weeks. In the per-protocol group, there was significant improvement in the International Prostate Symptom Score (IPSS), especially question numbers $1,3,4,5,6,7$, and the quality of life question $(\mathrm{P}=0.001, \mathrm{P}=0.007, \mathrm{P}<0.001, \mathrm{P}=0.003$, $\mathrm{P}=0.049, \mathrm{P}<0.001$, and $\mathrm{P}<0.001$, respectively). The Leeds sleep evaluation questionnaire (LSEQ) score for the sleep question improved from 64.36 to $70.43(\mathrm{P}=0.039)$. The NPi reduced from 0.4005 to $0.3573(\mathrm{P}=0.027)$; however, in many cases, there was no improvement in nocturnal polyuria itself. In intention-to-treat analysis, there were significant improvements in IPSS and LSEQ in 45 patients.

Conclusions: In elderly nocturnal polyuria patients, silodosin monotherapy exhibits good efficacy in improving nocturia and nocturnal polyuria; however, the mean NPi was still $>0.33$. Considering the high dropout rate of our study due to no implementation of 3-day frequency volume charts, prospective and large-scale studies are needed to confirm our results.

Keywords: Nocturia; Adrenergic alpha-Antagonists; Prostatic Hyperplasia

- Fund Support: This study was supported by the JW pharmaceutical company.

- Research Ethics: This study was approved by the local Ethics Committee and the Institutional Review Board of Chungbuk National University

Hospital (IRB No. 2013-03-006-005).

- Conflict of Interest: No potential conflict of interest relevant to this article was reported.

\section{INTRODUCTION}

Nocturnal polyuria (NP) is the most important cause of noctu- ria in elderly men, affecting up to $82 \%$ of patients with nocturia [1]. The elderly usually have many medical problems. A number of different medical problems and environmental factors

Corresponding author: Won Tae Kim (iD http://orcid.org/0000-0002-7482-4550 Department of Urology, Chungbuk National University College of Medicine, 1 Chungdae-ro, Seowon-gu, Cheongju 28644, Korea

E-mail: wtkimuro@chungbuk.ac.kr / Tel: +82-43-269-6133 / Fax: +82-43-269-6144 Submitted: June 3, 2015 / Accepted after revision: July 30, 2015

(1) 5 This is an Open Access article distributed under the terms of the Creative Commons Attribution Non-Commercial License (http://creativecommons.org/licenses/by-nc/3.0/) which permits unrestricted non-commercial use, distribution, and reproduction in any medium, provided the original work is properly cited. 
can cause NP [2]. Therefore, elderly men frequently suffer from nocturia, especially from NP [3]. Nocturia may give rise to frequent awakenings and sleep disorders in elderly patients $[4,5]$. The prevalence of lower urinary tract symptoms (LUTS) among Korean men aged $\geq 60$ years was $89.6 \%$, and the most common symptoms was nocturia (65.0\%) [6].

In the elderly, increased thirst and reduced levels of vasopressin are the main causes of larger nocturnal urine volume (NUV) [7]. In particular, disturbances of antidiuretic hormone secretion are a major cause of NP in elderly patients with benign prostatic hyperplasia (BPH) [4]. Generally, it is well documented that desmopressin is the first choice to treat NP [8]. However, desmopressin therapy has some limitations in clinical use for elderly patients because of its adverse effects, such as hyponatremia [9]. Thus, it is necessary to find alternatives to desmopressin for the management of NP in elderly $\mathrm{BPH}$ patients.

Recently, some studies have shown that tamsulosin is effective in improving nocturia and decreasing NUV in elderly BPH patients $[10,11]$. Similarly, other studies have reported that silodosin has a significant effect on nocturia [12-14]. However, although a-blockers have been effective in treating nocturia in BPH patients, the effectiveness of $\alpha$-blockers in reducing NUV in elderly men is controversial. Furthermore, there have been few studies on the effectiveness of silodosin for NP in elderly patients. In this study, we evaluated the effectiveness of silodosin in improving NP in elderly BPH patients with 3-day frequency volume charts.

\section{MATERIALS AND METHODS}

\section{Study Population and Data Collection}

Between April 2013 and April 2014, a prospective, multicenter study was performed in five hospital clinics. We enrolled BPH patients aged $\geq 60$ years, who were diagnosed with NP by using a 3-day frequency volume chart. Patients with a nocturnal polyuria index $(\mathrm{NPi})>0.33$ were included in the study. The following patients were excluded from the study: (1) those with conditions that could affect urinary function, such as a medication history within 1 month (patients treated with diuretics and a-blocker medications were required to undergo a 1-month washout period), neurological disease, sleep disorders (patients with insomnia or taking antipsychotics such as antidepressants and anxiolytic medications), urinary tract infections, prostate cancer, or global polyuria (24-hour urine volume that exceeded $40 \mathrm{~mL} / \mathrm{kg}$, as measured by using the frequency volume chart) and (2) younger age (under 60 years of age).
All patients were evaluated at the time of screening by using history taking, physical examination, prostate-specific antigen (PSA) tests, transrectal ultrasonography, uroflowmetry, the 3-day frequency volume chart, the International Prostate Symptom Score (IPSS), and the Leeds sleep evaluation questionnaire (LSEQ). At the time of screening, we did not recommend water restriction in order to minimize bias. After silodosin administration, patients were evaluated with the IPSS and the LSEQ at the 4-week follow-up, and the 3-day frequency volume chart, IPSS, and the LSEQ at the 12-week follow-up. The primary endpoint of this study was the mean change in NUV, and the secondary end-points were changes in nighttime frequency, IPSS, and LSEQ. A total of 54 patients who were diagnosed with NP $(\mathrm{NPi}>0.33)$ by using the initial 3-day frequency volume chart were enrolled in the study. Informed consent was obtained from each patient, and the study was approved by the local Ethics Committee and the Institutional Review Board of Chungbuk National University Hospital (IRB No. 2013-03-006-005).

\section{Definition of Variables}

The variables in the 3-day frequency volume chart were expressed as the average of the 3-day data. Nocturia was defined as an actual number of nighttime voids of $\geq 1$ according to the guidelines of the International Continence Society [15]. Generally, NP is defined as an NUV of more than $20 \%-33 \%$ of 24 hour urine volume, namely, an NPi of $\geq 0.20-0.33$ (percentage adjusted according to age: more than $20 \%$ of daily urine output at night in young patients and more than $33 \%$ in elderly patients) [15]. In this study, NP was defined as $\mathrm{NPi} \geq 0.33$ because all subjects were elderly patients over 60 years of age.

\section{The Questionnaires \\ International prostate symptom score}

Analysis of LUTS was based on responses to questions 1-7 of the IPSS questionnaire. Study participants were asked to choose among the six different answers, ranging from 0 (none) to 5 (5 or more). IPSS-voiding symptom (IPSS-V) was defined as the sum of scores for questions $1,3,5$, and 6 of the IPSS. IPSS-storage symptom (IPSS-S) was defined as the sum of scores for questions 2, 4, and 7 of the IPSS.

\section{Leeds sleep evaluation questionnaire}

The LSEQ is a standardized self-reporting instrument composed of ten 100-mm visual analogue questions that pertain to four items: the ease of getting to sleep (items 1-3), the quality of 
sleep (items 4 and 5), awakening from sleep (items 6 and 7), and behavior following wakefulness (items 8-10). The LSEQ is a sensitive indicator of subjectively felt changes in sleep latency and sleep quality. For each question, the subject responds by placing a vertical mark on the line $(0-100 \mathrm{~mm})$ to indicate his/ her present self-evaluation. The position of the mark indicates the nature and extent of the change, i.e., the middle of the line indicates no change, and the marker closer to the end of the line indicates larger changes (improvement on the left and worsening on the right) [16].

\section{Statistical analysis}

Differences between the two groups in terms of urodynamic and demographic variables were assessed using the MannWhitney $U$ test. The Wilcoxon signed-rank test was used to compare variables at screening and at the 12-week follow-up.
Intention-to-treat imputation of missing data was performed using the last-observation-carried-forward (LOCF) method. All statistical analyses were performed using IBM SPSS Statistics ver. 21.0 (IBM Co., Armonk, NY, USA). A P-value of $<0.05$ was considered statistically significant.

\section{RESULTS}

\section{Reasons for Dropout}

In total, 54 patients were enrolled in the study. Twenty-four patients dropped out. Five patients withdrew from the study due to a lack of efficacy for NP. Fifteen patients were lost to followup at 4 weeks ( 7 patients) and 12 weeks ( 8 patients). Three-day frequency volume charts at 12 weeks could not be collected from 4 patients. In the dropout group, 15 patients were visited at 4 weeks. Therefore, these patients were added to the intention

Table 1. Baseline characteristics of the total, ITT analysis, 12-week F/U, and non-F/U groups

\begin{tabular}{|c|c|c|c|c|c|}
\hline Characteristic & Total $(\mathrm{n}=54)$ & ITT group $(n=45)$ & 12-Week F/U group $(n=30)$ & Non-F/U group $(\mathrm{n}=24)$ & P-value \\
\hline Age (yr) & $68(64-72)$ & $68(63-72)$ & $67(63-72)$ & $68(65-74)$ & 0.459 \\
\hline PSA (ng/mL) & $1.02(0.49-1.80)$ & $0.97(0.42-1.78)$ & $1.08(0.43-1.83)$ & $0.92(0.53-1.83)$ & 0.821 \\
\hline Prostate size (g) & $27.7(21.6-33.8)$ & $27.1(20.1-35.1)$ & $26.8(20.5-36.1)$ & $28.2(21.8-31.5)$ & 0.94 \\
\hline Adenoma size (g) & $11.8(8.0-15.7)$ & $11.4(7.7-16.0)$ & $11.2(7.6-15.8)$ & $12.2(9.7-15.7)$ & 0.496 \\
\hline $\begin{array}{l}\text { Uroflowmetry } \\
\text { Qmax }(\mathrm{mL} / \mathrm{sec}) \\
\text { Qaverage }(\mathrm{mL} / \mathrm{sec}) \\
\text { Residual urine }(\mathrm{mL})\end{array}$ & $\begin{array}{c}11.5(8.5-14.7) \\
6.4(5.1-8.3) \\
18.5(0-48.7)\end{array}$ & $\begin{array}{c}11.0(8.5-16.5) \\
6.5(5.1-8.7) \\
17(0-49.5)\end{array}$ & $\begin{array}{c}11.1(8.4-14.7) \\
6.5(4.8-8.0) \\
10(0-34.8)\end{array}$ & $\begin{array}{c}12.0(10.3-15.5) \\
6.3(5.1-8.7) \\
25(0-51.7)\end{array}$ & $\begin{array}{l}0.401 \\
0.794 \\
0.238\end{array}$ \\
\hline $\begin{array}{l}\text { Voiding diary } \\
\text { 24-Hour urine volume }(\mathrm{mL}) \\
\text { Nocturnal urine volume }(\mathrm{mL}) \\
\text { NPi }\end{array}$ & $\begin{array}{c}1,868(1,640-2,187) \\
740(638-929) \\
39.0(34.3-42.8)\end{array}$ & $\begin{array}{c}1,860(1,608-2,225) \\
730(618-922) \\
38.8(34.0-43.8)\end{array}$ & $\begin{array}{l}1,837(1,609-2,232) \\
755(630-949) \\
39.0(34.0-44.3)\end{array}$ & $\begin{array}{c}1,929(1,650-2,262) \\
727(638-895) \\
38.6(33.7-41.3)\end{array}$ & $\begin{array}{l}0.814 \\
0.814 \\
0.338\end{array}$ \\
\hline $\begin{array}{l}\text { IPSS } \\
\text { Domain } 1 \\
\text { Domain } 2 \\
\text { Domain } 3 \\
\text { Domain } 4 \\
\text { Domain } 5 \\
\text { Domain } 6 \\
\text { Domain } 7 \\
\text { IPSS-V } \\
\text { IPSS-S } \\
\text { QoL }\end{array}$ & $\begin{array}{c}3(2-4) \\
3(2-4) \\
2(1-4) \\
3(1-5) \\
3(2-5) \\
2(0-3) \\
3(2-4) \\
10(6-15) \\
8(6-11) \\
4(3-5)\end{array}$ & $\begin{array}{c}3(1-4) \\
3(2-4) \\
2(1-4) \\
3(0-5) \\
3(2-5) \\
1(0-3) \\
3(2-4) \\
10(5-14) \\
9(6-11) \\
4(3-5)\end{array}$ & $\begin{array}{c}3(2-4) \\
3(2-4) \\
3(1-5) \\
3(0-5) \\
4(2-5) \\
2(0-3) \\
3(2-4) \\
11(6-15) \\
9(6-12) \\
4(3-5)\end{array}$ & $\begin{array}{l}3(1-4) \\
3(2-4) \\
2(1-3) \\
2(1-4) \\
3(2-5) \\
2(1-3) \\
3(2-4) \\
9(6-14) \\
8(6-11) \\
4(3-5)\end{array}$ & $\begin{array}{l}0.306 \\
0.465 \\
0.218 \\
0.436 \\
0.387 \\
0.872 \\
0.864 \\
0.346 \\
0.547 \\
0.837\end{array}$ \\
\hline LSEQ score & $67(51-74)$ & $69(53-76)$ & $70(54-76)$ & $63(50-74)$ & 0.363 \\
\hline
\end{tabular}

Values are presented as median (interquartile range).

ITT, intention to treat; F/U, follow-up; PSA, prostate-specific antigen; Qmax, maximum urinary flow rate; Qaverage, average urinary flow rate; NPi, nocturnal polyuria index; IPSS, international prostate symptom score; IPSS-V, IPSS-voiding symptoms; IPSS-S, IPSS-storage symptoms; QoL, quality of life; LSEQ, leeds sleep evaluation questionnaire.

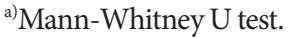


to treat (ITT) group.

\section{Baseline Characteristics of the Per-Protocol and Dropout Groups}

At screening, age, PSA, prostate size, uroflowmetry (maximum urinary flow rate, average urinary flow rate, and residual urine), 24-hour urine volume, NUV, NPi, each question of the IPSS, the IPSS-V, and the IPSS-S were not significantly different between the two groups. All the parameters are shown in Table 1. Fortysix of the total 54 patients were $\alpha$-blocker-naive patients, and in the per-protocol group, 28 patients were $\alpha$-blocker-naive patients.

\section{Changes in Parameters After Silodosin Administration at Screening and at 12-Week Follow-up in the Per-Protocol Group}

To evaluate the effects of silodosin on NP, we compared the variables from the 3-day frequency volume chart, the IPSS, and the LSEQ score at screening and 12 weeks after silodosin initiation. There was no significant change in 24-hour urine volume and daytime urine volume between screening and the 12-week follow-up $(\mathrm{P}=0.163$ and $\mathrm{P}=0.375$, respectively). NUV and NPi were significantly decreased at 12 weeks compared to screening $(\mathrm{P}=0.001$ and $\mathrm{P}=0.027$, respectively). For the IPSS, scores of questions $1,3,4,5,6$, and 7 were significantly decreased at 12 weeks compared to screening $(\mathrm{P}=0.001, \mathrm{P}=0.007, \mathrm{P}<0.001, \mathrm{P}=0.003, \mathrm{P}=0.049$, and $\mathrm{P}<0.001$, respectively). The score of question 2 was decreased at 12 weeks as compared to screening, although the difference was not statistically significant $(P=0.060)$. The scores of the IPSS- $V$ and IPSS-S were significantly decreased at 12 weeks compared to screening $(\mathrm{P}=0.001, \mathrm{P}<0.001$, respectively). The score of quality of life $(\mathrm{QoL})$ was significantly decreased at 12 weeks compared to screening $(\mathrm{P}<0.001)$. For the LSEQ, the overall scores were significantly increased at 12 weeks compared to screening $(\mathrm{P}=0.039)$ (Table 2).

\section{Changes in Parameters After Silodosin Administration at Screening and at Follow-up in the ITT Group}

To minimize bias, we performed an ITT analysis with the LOCF method. At the 4-week visit, patients only performed IPSS and LSEQ without a voiding diary. Therefore, there were no parameters of the 3-day frequency volume chart for the LOCF. For the IPSS, scores of all domains, including the IPSS-V and IPSS-S were significantly decreased at follow-up compared to screening $(\mathrm{P}=0.001, \mathrm{P}=0.020, \mathrm{P}=0.004, \mathrm{P}<0.001, \mathrm{P}=0.001, \mathrm{P}=0.038$, $\mathrm{P}<0.001, \mathrm{P}=0.001$, and $\mathrm{P}<0.001$, respectively). The QoL score was significantly decreased at follow-up compared to screening

Table 2. Changes in parameters after silodosin administration at screening and at a 12 -week F/U

\begin{tabular}{|c|c|c|c|c|c|c|}
\hline \multirow{2}{*}{ Variable } & \multicolumn{3}{|c|}{ Per-protocol group $(n=30)$} & \multicolumn{3}{|c|}{ ITT group $(\mathrm{n}=45)$} \\
\hline & Screening & 12-Week F/U & P-value ${ }^{a)}$ & Screening & $\mathrm{F} / \mathrm{U}$ & P-value \\
\hline \multicolumn{7}{|l|}{ Voiding diary } \\
\hline 24 -Hour urine volume $(\mathrm{mL})$ & $1,837(1,609-2,232)$ & $1,768(1,464-2,238)$ & 0.163 & - & - & - \\
\hline Daytime urine volume (mL) & $1,094(930-1,377)$ & $1,170(865-1,512)$ & 0.375 & - & - & - \\
\hline Nocturnal urine volume $(\mathrm{mL})$ & 755 (630-949) & $650(458-913)$ & 0.001 & - & - & - \\
\hline $\mathrm{NPi}$ & $39.0(34.0-44.3)$ & $34.9(28.5-44.7)$ & 0.027 & - & - & - \\
\hline \multicolumn{7}{|l|}{ IPSS } \\
\hline Domain 1 & $3(2-4)$ & $1(0-3)$ & 0.001 & $3(1-4)$ & $2(0-3)$ & 0.001 \\
\hline Domain 2 & $3(2-4)$ & $2(1-3)$ & 0.060 & $3(2-4)$ & $2(1-3)$ & 0.020 \\
\hline Domain 3 & $3(1-5)$ & $1(0-3)$ & 0.007 & $2(1-4)$ & $1(0-2)$ & 0.004 \\
\hline Domain 4 & $3(0-5)$ & $1(0-2)$ & $<0.001$ & $3(0-5)$ & $1(0-3)$ & $<0.001$ \\
\hline Domain 5 & $4(2-5)$ & $2(1-4)$ & 0.003 & $3(2-5)$ & $2(1-4)$ & 0.001 \\
\hline Domain 6 & $2(0-3)$ & $1(0-3)$ & 0.049 & $1(0-3)$ & $1(0-3)$ & 0.038 \\
\hline Domain 7 & $3(2-4)$ & $2(1-3)$ & $<0.001$ & $3(2-4)$ & $2(2-3)$ & $<0.001$ \\
\hline IPSS-V & $11(6-15)$ & $6(3-11)$ & 0.001 & $10(5-14)$ & $6(3-10)$ & $<0.001$ \\
\hline IPSS-S & $9(6-12)$ & $6(4-9)$ & $<0.001$ & $9(6-11)$ & $6(4-9)$ & $<0.001$ \\
\hline QoL & $4(3-5)$ & $3(2-4)$ & $<0.001$ & $4(3-5)$ & $3(2-4)$ & $<0.001$ \\
\hline LSEQ score & $70(54-76)$ & $75(56-87)$ & 0.039 & $69(53-76)$ & $70(56-81)$ & 0.024 \\
\hline
\end{tabular}

Values are presented as median (interquartile range).

F/U, follow-up; ITT, intention to treat; NPi, nocturnal polyuria index; IPSS, international prostate symptom score; IPSS-V, IPSS-voiding symptoms; IPSS-S, IPSS-storage symptoms; QoL, quality of life; LSEQ, leeds sleep evaluation questionnaire.

${ }^{a)}$ Wilcoxon signed-rank test. 
$(\mathrm{P}<0.001)$. For the LSEQ, the overall scores were significantly increased at follow-up compared to screening $(\mathrm{P}=0.024)$ (Table 2).

\section{DISCUSSION}

In this study, we found that NP improved 12 weeks after silodosin initiation. This study also showed that silodosin administration improved sleep quality in NP patients at 12 weeks of follow-up. This is the first study evaluating the effect of silodosin on NP with 3-day frequency volume charts.

$\mathrm{NP}$ is one of the most important causes of nocturia in the elderly group [17]. Nocturia may result from various causes, including pathologic disease, cardiovascular disease, environmental factors, and sleep disorders [18-20]. Among these causes, $\mathrm{BPH}$ is a potential risk factor for nocturia, and about $40 \%$ of the elderly men over 50 years of age suffer from $\mathrm{BPH}$ [20].

In a randomized, placebo-controlled, double-blind phase 3 study, silodosin was effective for nocturia in men with LUTS suggestive of $\mathrm{BPH}$ [13]. A total of 1,479 patients were treated with silodosin or placebo, and men treated with silodosin showed a significant improvement in nocturia symptoms (53.4\% vs. $42.8 \%, \mathrm{P}<0.0001$ ). Of the patients with $\geq 2$ nocturnal voids at baseline, $61 \%$ treated with silodosin had a reduction of $<1$ voids/night $(\mathrm{P}=0.0003)$; patients treated with silodosin had a higher frequency of $<2$ nocturia at the completion of the study than those treated with placebo $(29.3 \%$ vs. $19.0 \%$; $\mathrm{P}=0.0002$ ). Silodosin consistently and significantly improves nocturia in men with LUTS/BPH [13]. Silodosin is also effective for improving nocturia in patients taking $\alpha$-blockers [12]. We also showed that silodosin reduced nocturia significantly. Thus, for $a$-blocker users, silodosin could be an appropriate drug for treating nocturia.

Nocturia is a major cause of sleep impairment [7]. Generally, sleep influences nocturnal urine output because the amount of urine produced decreases at night [21]. Elderly patients with frequent awakenings tend to have a larger NUV than those with fewer awakenings [4]. Sleep quality can be improved by reducing nocturia [7]. We showed that silodosin administration improved sleep quality and alleviated nocturia in elderly BPH patients with NP.

Interference with endogenous production of the arginine vasopressin (AVP) hormone, excessive production of atrial natriuretic peptides (notably associated with sleep apnea and chronic heart failure), and external factors, such as diuretics or fluid intake at night, are important clinical conditions causing NP [22]. Desmopressin acetate is effective in treating polyuric conditions, such as primary nocturnal enuresis and central diabetes insipidus [23]. Thus, desmopressin is the most commonly prescribed medication to treat nocturia with NP. Wang et al. [8] reported that desmopressin leads to a significant decrease in nocturia and NUV.

However, desmopressin therapy has some limitations in clinical use. First, desmopressin is more likely to be effective in patients with NP due to insufficient secretion of AVP because it is a synthetic analog of AVP. Second, patients taking desmopressin have a potential risk of developing hyponatremia [9]. Van Kerrebroeck et al. [19] reported that among 184 patients who experienced hyponatremia, 17 had borderline hyponatremia (serum sodium level $<130 \mathrm{mmol} / \mathrm{L}$ ), and $20 \mathrm{had}$ a postbaseline serum sodium level below normal. Weiss et al. [17] recommended that $50-\mu \mathrm{g}$ desmopressin is the minimum effective and safe dose for use in men with nocturia and NP. They also indicated that close monitoring of serum electrolytes is necessary to prevent the occurrence of hyponatremia. For these reasons, adverse events of desmopressin, such as hyponatremia, are serious obstacles to long-term clinical use and high doses of desmopressin in elderly patients.

In recent years, some studies showing the efficacy of $\alpha 1$ adrenergic receptor antagonists for the treatment of $\mathrm{BPH}$ patients with NP have been reported $[10,24]$. In a long-term, prospective study, it was found that tamsulosin treatment decreased the mean nighttime frequency and NUV, and increased maximum voided volume and daytime urine volume in $\mathrm{BPH}$ patients with NP [10]. The authors of these studies suggested that long-term tamsulosin treatment might reduce nighttime urine production by correcting disturbances of the circadian regulation of urine production in BPH patients with NP.

In the current study, we also found that silodosin treatment decreased NUV and NPi at 12 weeks. These results suggest that silodosin could be a useful drug for managing NP in elderly $\mathrm{BPH}$ patients without serious adverse events. However, the mean NPi was not decreased to lower than 0.33. Silodosin, therefore, did not improve NP. Although NP occurs due to various causes, NP in elderly BPH patients seems to be primarily caused by a large NUV. Thus, we suggest that silodosin can be an alternative option to treat $\mathrm{NP}$ and nocturia in elderly $\mathrm{BPH}$ patients with a risk of hyponatremia before trying desmopressin. Of course, additional randomized, double-blind comparative studies comparing the efficacy of silodosin versus other 
a-blockers for treating nocturia and NP should be performed.

There were several weaknesses to this study. First, the small study population due to a high dropout rate is a potential weakness. Larger-scale cohort studies are needed to confirm our results. Second, this study was not a randomized controlled trial (RCT) due to placebo drug problems. Therefore, the level of evidence provided by this study is lower compared to an RCT. Third, the dropout rates of this study were very high. However, the causes of follow-up loss may actually reduce the risk of bias and strengthen the results of this study. Fourth, we could not identify the mechanism whereby silodosin decreased the NUV. Further studies to elucidate the exact mechanism are necessary.

In conclusion, silodosin monotherapy exhibits good efficacy in improving nocturia and NP (although the NPi remained $>0.33$ ) in elderly NP patients. However, considering the high dropout rate due to failure to complete 3-day frequency volume charts, prospective, large-scale studies are needed to confirm our results.

\section{REFERENCES}

1. Chang SC, Lin AT, Chen KK, Chang LS. Multifactorial nature of male nocturia. Urology 2006;67:541-4.

2. Cornu JN, Abrams P, Chapple CR, Dmochowski RR, Lemack GE, Michel MC, et al. A contemporary assessment of nocturia: definition, epidemiology, pathophysiology, and management: a systematic review and meta-analysis. Eur Urol 2012;62:877-90.

3. Van Dijk MM, Wijkstra H, Debruyne FM, De La Rosette JJ, Michel $\mathrm{MC}$. The role of nocturia in the quality of life of men with lower urinary tract symptoms. BJU Int 2010;105:1141-6.

4. Asplund R. Nocturia, nocturnal polyuria, and sleep quality in the elderly. J Psychosom Res 2004;56:517-25.

5. Middelkoop HA, Smilde-van den Doel DA, Neven AK, Kamphuisen HA, Springer CP. Subjective sleep characteristics of 1,485 males and females aged 50-93: effects of sex and age, and factors related to self-evaluated quality of sleep. J Gerontol A Biol Sci Med Sci 1996;51:M108-15.

6. Kim TH, Han DH, Lee KS. The prevalence of lower urinary tract symptoms in Korean men aged 40 years or older: a populationbased survey. INJ 2014;18:126-32.

7. Asplund R, Aberg H. Health of the elderly with regard to sleep and nocturnal micturition. Scand J Prim Health Care 1992;10:98-104.

8. Wang CJ, Lin YN, Huang SW, Chang CH. Low dose oral desmopressin for nocturnal polyuria in patients with benign prostatic hyperplasia: a double-blind, placebo controlled, randomized study. J
Urol 2011;185:219-23.

9. Delfanian K, Zawada ET Jr. DDAVP-associated hyponatremia. S D J Med 2001;54:255-6.

10. Kojima Y, Sasaki S, Imura M, Kubota Y, Hayashi Y, Kohri K. Tamsulosin reduces nighttime urine production in benign prostatic hyperplasia patients with nocturnal polyuria: a prospective open-label long-term study using frequency-volume chart. Neurourol Urodyn 2012;31:80-5.

11. Yoshida M, Inadome A, Masunaga K, Nagata T, Yoshiyasu T. Effectiveness of tamsulosin hydrochloride and its mechanism in improving nocturia associated with lower urinary tract symptoms/ benign prostatic hyperplasia. Neurourol Urodyn 2010;29:1276-81.

12. Chapple CR, Montorsi F, Tammela TL, Wirth M, Koldewijn E, Fernández Fernandez E, et al. Silodosin therapy for lower urinary tract symptoms in men with suspected benign prostatic hyperplasia: results of an international, randomized, double-blind, placeboand active-controlled clinical trial performed in Europe. Eur Urol 2011;59:342-52.

13. Eisenhardt A, Schneider T, Cruz F, Oelke M. Consistent and significant improvement of nighttime voiding frequency (nocturia) with silodosin in men with LUTS suggestive of BPH: pooled analysis of three randomized, placebo-controlled, double-blind phase III studies. World J Urol 2014;32:1119-25.

14. Takao T, Tsujimura A, Kiuchi H, Takezawa K, Nonomura N, Miyagawa Y. Improvement of nocturia and sleep disturbance by silodosin in male patients with lower urinary tract symptoms. Int J Urol 2015;22:236-8.

15. van Kerrebroeck P, Abrams P, Chaikin D, Donovan J, Fonda D, Jackson S, et al. The standardisation of terminology in nocturia: report from the Standardisation Sub-committee of the International Continence Society. Neurourol Urodyn 2002;21:179-83.

16. Tarrasch R, Laudon M, Zisapel N. Cross-cultural validation of the Leeds sleep evaluation questionnaire (LSEQ) in insomnia patients. Hum Psychopharmacol 2003;18:603-10.

17. Weiss JP, Herschorn S, Albei CD, van der Meulen EA. Efficacy and safety of low dose desmopressin orally disintegrating tablet in men with nocturia: results of a multicenter, randomized, double-blind, placebo controlled, parallel group study. J Urol 2013;190:965-72.

18. Montorsi F, Mercadante D. Diagnosis of BPH and treatment of LUTS among GPs: a European survey. Int J Clin Pract 2013;67:1149.

19. van Kerrebroeck P, Rezapour M, Cortesse A, Thuroff J, Riis A, Norgaard JP. Desmopressin in the treatment of nocturia: a doubleblind, placebo-controlled study. Eur Urol 2007;52:221-9.

20. Yoshimura K, Ohara H, Ichioka K, Terada N, Matsui Y, Terai A, et 
al. Nocturia and benign prostatic hyperplasia. Urology 2003;61:78690.

21. Thomas S. Some effects of changes of posture on water and electrolyte excretion by the human kidney. J Physiol 1957;139:337-52.

22. Gulur DM, Mevcha AM, Drake MJ. Nocturia as a manifestation of systemic disease. BJU Int 2011;107:702-13.

23. Ahmed AF, Amin MM, Ali MM, Shalaby EA. Efficacy of an enure- sis alarm, desmopressin, and combination therapy in the treatment of saudi children with primary monosymptomatic nocturnal enuresis. Korean J Urol 2013;54:783-90.

24. Takahashi S, Tajima A, Matsushima H, Kawamura T, Tominaga T, Kitamura T. Clinical efficacy of an alpha1A/D-adrenoceptor blocker (naftopidil) on overactive bladder symptoms in patients with benign prostatic hyperplasia. Int J Urol 2006;13:15-20. 